\title{
What Are the Effects of
} Self-Regulation Phases and Strategies for Chinese Students? A Meta-Analysis of Two Decades Research of the Association Between Self-Regulation and Academic Performance

\author{
Junyi $\mathrm{Li}^{1,2}$, Hui Ye ${ }^{2}$, Yun Tang ${ }^{2,3,4 *}$, Zongkui Zhou ${ }^{2,3,4}$ and Xiangen $\mathrm{Hu}^{2,3,5}$ \\ ${ }^{1}$ School of Teacher Education and Psychology, Sichuan Normal University, Chengdu, China, ${ }^{2}$ School of Psychology, Central \\ China Normal University, Wuhan, China, ${ }^{3}$ Central China Normal University Branch, Collaborative Innovation Center of \\ Assessment toward Basic Education Quality, Wuhan, China, ${ }^{4}$ Key Laboratory of Adolescent Cyberpsychology and Behavior, \\ Ministry of Education, Wuhan, China, ${ }^{5}$ Institute for Intelligent Systems, University of Memphis, Memphis, TN, United States
}

OPEN ACCESS

Edited by:

Jesus de la Fuente,

Universidad de Navarra, Spain

Reviewed by:

Jesús Nicasio García Sánchez,

Universidad de León, Spain

Giovanna Bubbico,

Department of Neuroscience, Imaging and Clinical Sciences, University of "G.

d'Annunzio" Chieti - Pescara, Italy

${ }^{*}$ Correspondence:

Yun Tang

tangyun@mail.ccnu.edu.cn

Specialty section:

This article was submitted to

Educational Psychology,

a section of the journal

Frontiers in Psychology

Received: 08 March 2018 Accepted: 19 November 2018 Published: 18 December 2018

Citation:

Li J, Ye H, Tang Y, Zhou Z and HuX (2018) What Are the Effects of Self-Regulation Phases and Strategies for Chinese Students? A Meta-Analysis of Two Decades Research of the Association Between Self-Regulation and Academic Performance. Front. Psychol. 9:2434. doi: 10.3389/fpsyg.2018.02434
Background: Self-regulated learning refers to the monitoring and controlling of one's own cognitive performance before, during, and after a learning episode. Previous literature suggested that self-regulated learning had a significant relationship with academic achievement, but not all self-regulated learning strategies exerted the same influences. Using an invalid strategy may waste the limited psychological resources, which will cause the ego depletion effect. The present meta-analysis study intended to search for the best self-regulated learning strategies and inefficient strategies for Chinese students in elementary and secondary school, and analyzed the critical phases of self-regulated learning according to Zimmerman's theory. The moderating effects of gender, grade, and publication year were also analyzed.

Methods: Empirical studies which conducted in real teaching situations of elementary and secondary education were systematically searched using Chinese academic databases. Studies focused on undergraduate students, students of special education, or online learning environments were excluded. Fifty-five cross-sectional studies and four intervention studies (which generated 264 independent samples) were included with a total sample size of 23,497 participants. Random effects model was chosen in the current meta-analysis, and publication bias was also examined.

Results: The results indicated that the overall effect size of self-regulated learning on academic achievement was small for primary and secondary school students in China. The effect sizes of self-efficacy, task strategies, and self-evaluation were relatively higher than other strategies. Self-regulated learning strategies have the largest effect size on science disciplines (including mathematics and physics). Performance phase and self-reflection phase are key phases of self-regulated learning. From 1998 to 2016, the effect size between self-regulated learning and academic achievement was gradually decreasing. 
Conclusions: The main findings of the current study showed that self-efficacy, task strategies, and self-evaluation were key self-regulated learning strategies for Chinese students. Performance phase and self-reflection phase played significant roles in the process of self-regulated learning. Future studies need to include more intervention studies with rigorous treatment fidelity control and provide more empirical evidence from online learning, so as to compare the different effects of self-regulated learning between traditional education and online education.

Keywords: self-regulated learning, academic achievement, elementary and secondary education, cross-sectional studies, meta-analysis

\section{INTRODUCTION}

"How can one become a professional learner?" is one of the most important topics in educational psychology. Professional learners are good at using strategies to help their study and holding openness view to difficult tasks, and they are willing to accept challenges until they reach the goal. Using effective learning strategies, having strong self-efficacy and will power are significant characteristics of professional learners (Sternberg and Williams, 2010), all of which are characteristics of selfregulated learning (SRL). Unlike measures of mental ability or academic performance skill, self-regulated learning refers to the self-directive processes and self-beliefs that enable learners to transform their mental abilities into an academic performance skill (Zimmerman, 2008). Self-regulated learning also has been highly praised as the key competence to initiate and maintain lifelong learning (EU Council, 2002). Self-regulated learning could have a wide influence in areas, including subjective well-being, physical health, social achievement, economy, and online education (Mischel et al., 2010; Kizilcec et al., 2017). In education, researchers have found that self-regulated learning has a significant association with performance self-efficacy, learning motivation, and conscientiousness (Pintrich, 2004; FernandezRio et al., 2017). These important traits also promote academic performance (Everaert et al., 2017; Street et al., 2017; Pascoe et al., 2018). Thus, the relationship between self-regulated learning and academic performance have attracted much attention.

\section{SELF-REGULATED LEARNING STRATEGIES AND ACADEMIC PERFORMANCE}

In Western countries, researchers have proved the effectiveness of self-regulated learning on academic achievement (Paris and Paris, 2001; Dignath et al., 2008; Sadati and Simin, 2017) and learning motivation (Pintrich, 1999). In China, Liu and Chen (2000) found that online self-regulated learning positively predicted writing scores of undergraduate students. Li et al. (2015) suggested that time value, time monitor, and selfefficacy have significant association with academic achievement in elementary school students. In secondary school, the effectiveness of self-regulated learning on academic achievement has also been verified (Chen and Hu, 2008; Zhang et al., 2012a).
Therefore, there is a similar effect to Western education on the relationship between self-regulated learning and academic performance.

Self-regulated learning consists of many strategies, including goal setting, self-efficacy, goal orientation, metacognitive monitoring, self-evaluation and so on (Panadero, 2017). As an integrated conception, self-regulation promotes academic performance, but meta-analysis study indicated that not all self-regulated strategies are effective. Specifically, rehearsal learning, organization, and peer learning were found not significantly associated with GPA (Richardson et al., 2012). To explain the lack of effectiveness, Baumeister et al. (1994) proposed the strength model of self-control which suggested that psychological resource could be consumed by using selfregulated strategies. If the preceding self-regulated strategy consumes too much psychological resource, the performance of the subsequent strategy would decrease, leading to ego depletion (Li et al., 2011a). These results indicated that it is necessary to find out the most effective strategies for different students or teaching environments, so that we can save psychological resources and promote academic performance to the greatest extent. Thus, the first goal of the present study was to identify the best self-regulated learning strategy and invalid strategy for Chinese students in primary and secondary school.

\section{PHASES AND SUB-PROCESSES OF SELF-REGULATION}

Self-regulated learning is a multidimensional construct that emphasizes the active role of the learner (Panadero, 2017). There are many definitions of self-regulation, and scholars did not reach a consensus in different area, even within educational psychology (Li et al., 2011a; Zimmerman, 2008). For example, the distinction between self-regulated learning and metacognitive learning strategies is often a fuzzy one that lacks clarification (Dignath et al., 2008). Metacognition and self-regulation sometimes refer to the same concept, whereas other models view metacognitive strategies as an important element of self-regulation. The terminology of self-regulation is also confounding, referred to as self-control, self-management, or metacognition in different areas. Hence, a comprehensive theory for SRL is needed to settle this problem. In the present study, we turned to the cyclical model of SRL proposed by Zimmerman (2008). It assumes 
bidirectional relationships of SRL processes across three phases, namely, forethought phase, performance phase (online SRL), and self-reflection phase (offline SRL). There is growing empirical evidences for this model: studies have indicated that students' self-efficacy was positively correlated with academic achievement (Chen, 2011; Doménechbetoret et al., 2017; Street et al., 2017). Microanalyses of self-regulated processes and sources of motivation have been used most frequently to investigate learning of athletic skills, such as free-throw shooting, volleyball serving and dart throwing, and these measures of SRL revealed significant differences between experts, non-experts, and novices (Zimmerman, 2008; Cleary and Zimmerman, 2010). When compared to non-experts and novices, experts made the most extensive use of SRL processes and reported the most positive motivational beliefs and feelings (Kitsantas and Zimmerman, 2002). Furthermore, research showed that novices who were taught multiple strategies displayed significantly greater athletic skill and improved motivational beliefs during relatively brief practice sessions than novices in a control group (Cleary et al., 2006). These evidences indicated that Zimmerman's model could distinguish between novices and experts effectively, it also made a good synthesis and elaboration of otherwise confusing SRL strategies, which has been supported by relevant empirical studies. Consequently, the second goal of the present study was to explore the effect sizes of sub-processes of self-regulation (i.e., forethought phase, performance phase, and self-reflection phase) according to Zimmerman's model in Chinese school settings, so as to find the detailed relationship of each phase on academic achievement.

\section{POTENTIAL MODERATORS BETWEEN SRL AND ACADEMIC ACHIEVEMENT}

Most studies on SRL in school has been conducted with older students, because younger students in elementary school probably have difficulties with SRL (Paris and Newman, 1990; Zimmerman, 1990; Panadero, 2017). Take students' views of their own abilities and motivation as an example. Young children sometimes focus on the wrong criteria of ability and exaggerate the available evidence. They often confuse perceptions of academic ability with appropriate social behavior (Stipek and Tannatt, 1984). For example, researchers found that first-grade students believe that sharing distinguishes average and smart students and that children who receive a great deal of criticism are less able than their peers (Blumenfeld et al., 1982). Studies also revealed that compared with 11-12 years old students, 7-8 years olds relatively less reflected on their performance and seldom evaluated and controlled their cognitive abilities (Paris and Newman, 1990). Although some studies showed that preschool children already started to use SRL strategies, and young children do engage in activities to self-regulate their learning (Perry et al., 2002, 2004; Schneider and Lockl, 2002), the level of SRL of younger students could be different from that of older students.

In addition, there is empirical evidence illustrate that girls outperform boys in adjusting to school (Ortiz and Bornacelly, 2017), whereas the gender difference in students' self-regulation competence may be one of the important factors for girls' advantage (Jiao and Gai, 2011). Girls' selfcontrol is significantly higher than that of boys at 2, 4, and 11 years old (Zhang et al., 2012b). A meta-analytic study about children's performance at the task of resisting the temptation have found that the performance of girls was better than boys (Silverman, 2002). From the insight of academic achievement, the motivations and emotions of girls and boys toward mathematics were probably significantly different. In K12 education, girls reported increasingly more negative attitudes toward mathematics performance and showed more selfderogating attributions about their mathematics performance (Royer and Walles, 2007; Hyde et al., 2008). In early adolescence, gender differences have also been found for mathematics selfconcept and mathematics utility (Eccles et al., 1993). These evidences suggested that the relationship between SRL and academic achievement may be moderated by gender.

Finally, the year of publication is an interesting variable, considering the rapid growth of economy and technology in China from 1990s to 2010s. To our knowledge, few studies of meta-analysis have explored the moderating effect of publication year by using meta-regression. This type of analysis could allow us to observe the hidden changing patterns of target variables, such as the significant differences of cohort (e.g., post-80s and post-00s) on the relationship between SRL and academic achievement. Thus, it is intriguing to examine if publication year would be a moderator in the meta-analysis.

\section{WHY CHINESE STUDENTS?}

Although researchers have provided critical reviews of existing self-regulated learning studies (e.g., Panadero, 2017; Sahdan and Abidin, 2017), they have rarely included studies from outside Europe or North America (Richardson et al., 2012). In fact, because of language issues, few studies conducted in Asia were located and Asian effect sizes were omitted from the meta-analysis. In addition, education does not take place in a vacuum, the results of education will naturally differ with different culture in different environments. Many studies have found students in East Asia outperformed their counterparts in the West in mathematic achievement (Mullis et al., 2000, 2004; Jerrim and Shure, 2016). However, teaching methods in East Asia are not perceived as advanced as in Western countries (Leung et al., 2006). For example, China is very often content oriented and examination driven. Large class size is the norm, and classroom teaching is often conducted in a whole class setting. The parity between the high math performance and a lack of advanced teaching methods is puzzling (Leung et al., 2006). It prompts for a call for further studies about the learning process of Chinese students, such as how they use self-regulated strategies to help their study. Hence, the present study includes empirical self-regulated learning studies from China (published in Chinese journals), attempting to provide an Asian insight in this field, and to understand the relationship between selfregulated learning and academic achievement of elementary and secondary education in China. 


\section{RESEARCH QUESTIONS AND HYPOTHESES}

Based on the reasons mentioned above, we proposed several research questions in the present study. First, what are the most and least effective self-regulated learning strategies for Chinese students? Second, according to Zimmerman's cyclical model of self-regulated learning, which SRL phase has the largest effect size on academic achievement? Third, are the moderating effects of gender, educational stage, and publication year significant? How do they impact the relationship between self-regulated learning and academic achievement? The following hypotheses were proposed.

1. The effect size of SRL on academic achievement would be significant for primary and secondary school students in China.

2. Significant differences would be found for the effect sizes between different phases and sub-processes of SRL.

3. The relationship between SRL and academic achievement in high school students may be stronger than that of elementary school students.

4. The effect sizes of SRL on academic achievement in girls are larger than those of boys.

\section{METHODS}

\section{Literature Search}

The present meta-analysis aimed to get conclusions from real teaching situations in China, and only Chinese journals were considered to narrow down the focus. Empirical studies were searched using the CNKI (China National Knowledge Infrastructure), Wanfang Database, and Vip Paper Check System. The following key words were used: "Self-regulated learning," "self-monitoring," "self-management," "time management," "learning strategies," "metacognition," "goal orientation," "goal setting," "self-efficacy," "self-reflection," "self-evaluation"; "academic achievement," "interim/final grade," "academic performance," "grades," and "academic success." Then, we also searched through the reference lists of systematic review articles of SRL.

\section{Inclusion and Exclusion Criteria}

After literature searching, all the authors discussed the following inclusion and exclusion criteria: (1) In order to ensure ecological validity, empirical studies should be conducted in real teaching situations. (2) Participants in the empirical studies must be elementary, junior high, or senior high school students in China. Undergraduate students and students of special education were excluded. (3) Empirical studies based on the online learning environments were excluded. (4) Empirical studies should report the correlation coefficients, sample sizes, means, and standard deviations. After literature screening (See Figure 1), 59 empirical studies (i.e., 55 cross-sectional studies and 4 intervention studies) were collected (Detailed information of these studies can be found in the Appendix).

\section{Coding of Studies}

We coded the studies and computed effect size using Comprehensive Meta-Analysis version 2 (Borenstein et al., 2005). The following information from each paper were coded: (1) basic information (title of the study, publication year and journal); (2) type of SRL strategies; (3) type of academic achievement, including language performance (Chinese and English), science performance (mathematics and physics), and integrated performance (i.e., when the study did not specify disciplines but use integrated interim or final grades); (4) educational stage, including elementary school, junior high school, and senior high school; (5) proportion of female; (6) phase of SRL, which was classified into forethought phase, performance phase, or self-reflection phase according to Zimmerman's (2008) cyclical model of SRL. For the crosssectional studies (55 studies), the correlation coefficient and sample size were extracted to calculate the effect size. As for the intervention studies (only 4 studies), sample sizes, means, and standard deviations in experimental group and control group were extracted.

All articles were separately coded by the first author and the second author. Inter-rater agreement CA $=2^{*}(59-14) /(59+59)$ $=0.763$. Because the two authors had different opinions about the classification of SRL strategies, they did not reach a consensus on 14 of the 59 studies. In cases where classification was not in agreement, the two authors discussed and came to a consensus.

\section{Fidelity of Intervention Studies}

Although most studies in the current meta-analysis were cross-sectional studies, 4 out of the 59 studies were based on intervention. It is necessary to report treatment fidelity of these intervention studies to ensure that study outcomes were due to treatment rather than factors incidental to the intervention (Díaz-Prieto and García-Sánchez, 2015; CanedoGarcía et al., 2017). Table 1 showed the treatment fidelity of the four intervention studies in the meta-analysis. Specifically, one study trained intervention providers. Two studies had clear intervention protocol. One study used interview to check the efficacy of intervention. Pre-post measures were used by three studies. All studies have the record of the sessions.

\section{Effect Size and Publication Bias}

Most empirical studies reported correlations, so the present meta-analysis used $r$ to indicate effect size. Then we transformed $r$ into Cohen's $d$ (i.e., standardized mean difference). A few studies reported the means, sample sizes, and standard deviations of experimental group and control group, we also transformed these number into Cohen's $d$. In interpreting the effect size, we followed Rosenthal (1996)'s guidelines $(d=0 \sim 0.29$, small; $d=0.30$ $\sim 0.79$, medium, $d=0.80 \sim 1.29$, large). The current metaanalysis was conducted with a random effects model, because accumulated evidence suggested heterogeneity in effect sizes (National Research Council, 1992), and we wished to draw inferences beyond the set of studies included in the analysis (Hedges and Vevea, 1998; Borenstein et al., 2010).

Journals tend to accept articles that report significant results, which may probably cause publication bias. We conducted 


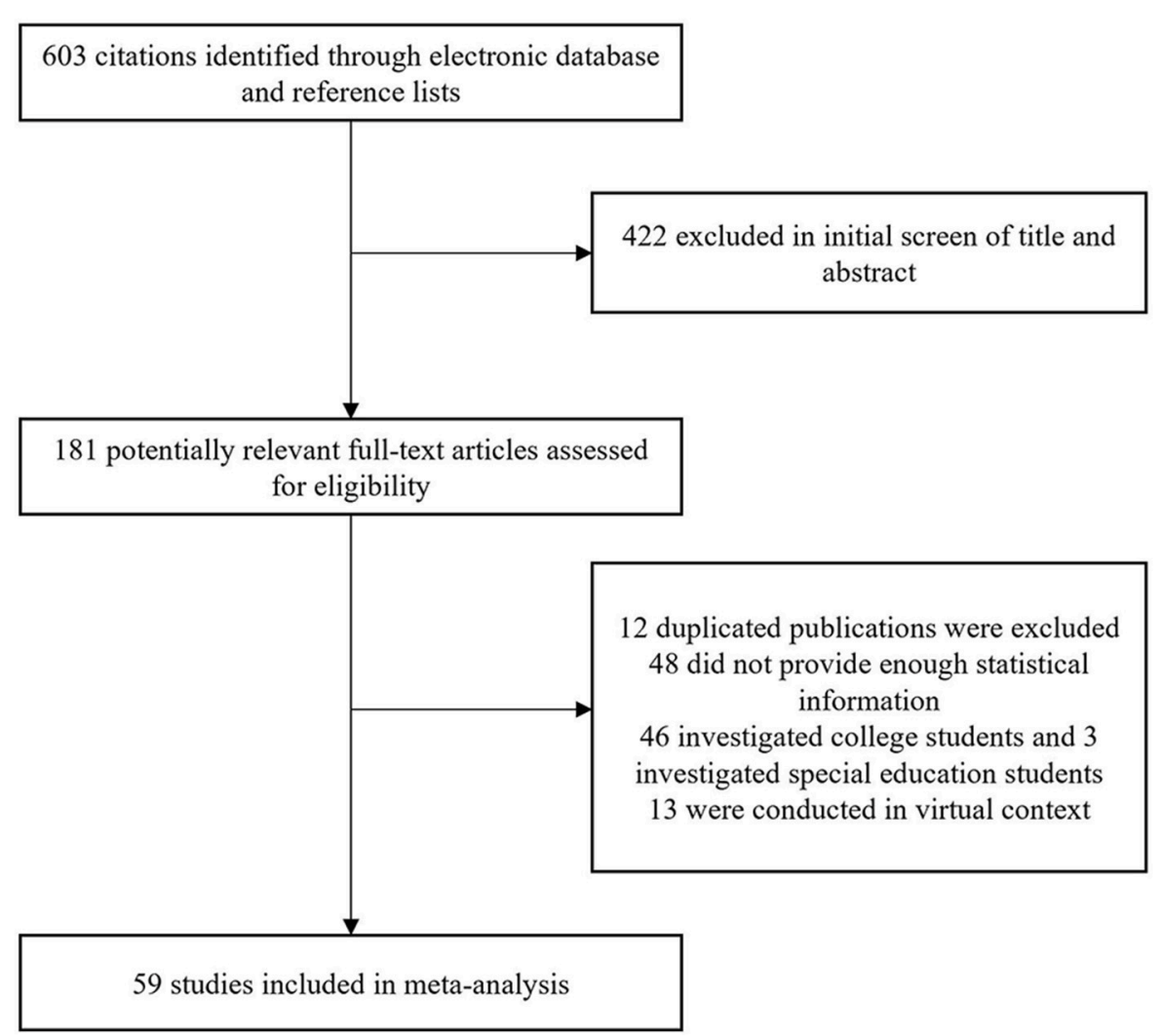

FIGURE 1 | Flow diagram of papers included in the present study.

analyses to address the possibility that the results might be affected by publication bias. Funnel plot, fail-safe $N$, and trim and fill procedures were used to examine publication bias. The horizontal axis of funnel plot stands for effect size, and the vertical axis of funnel plot stands for standard error. In the presence of publication bias, the bottom of the plot would tend to show a higher concentration of studies on one side of the mean than the other. Fail-safe $N$ computes the number of missing studies that would need to be added to the analysis to yield a statistically nonsignificant overall effect. Rothstein et al. (2006) suggested if failsafe $N$ is less than $5 k+10$, meta-analysis would exist publication bias. Trim and fill procedures were implemented to impute studies on the funnel plot and keep the plot symmetric (Duvall and Tweedie, 2000).

\section{RESULTS}

\section{SRL Strategies and Academic Achievement}

Two hundred and sixty-three independent samples reported the correlations between specific SRL strategies (e.g., attribution or goal setting) and academic achievement. Five independent samples viewed SRL as an integrated conception and reported the correlations between SRL and academic performance. Table 2 displayed the effect sizes of ten SRL strategies. The effect sizes of self-efficacy, self-evaluation, and task strategies were relatively larger than other strategies. The effect sizes of attribution and goal orientation were relatively small. Given that the sample size of goal setting, integrated (i.e., the independent samples that did not distinguish specific SRL strategy), and self-satisfaction were smaller than 8 (Borenstein et al., 2009), further discussion about these strategies were omitted. When all the empirical studies were pulled together, the effect size of SRL was 0.435 . Heterogeneity test found that there was considerable heterogeneity across the independent samples $\left[Q_{(263)}=3,766.115, p<0.001, I^{2}=93.0 \%\right]$, suggesting the presence of moderators.

Table 3 shows the mean effect sizes grouped according to disciplines. Specifically, the effect size of science (including performances of mathematics and physics) was relatively higher than that of language (including performances of Chinese and English). There were many empirical studies did not specify disciplines, but used integrated interim or final grades to measure students' academic achievement. These independent samples were labeled as integrated. The mean effect size of the integrated performance was 0.592 .

\section{Phases of SRL and Academic Performance}

According to Zimmerman's theory, we classified SRL strategies into forethought phase, performance phase, and self-reflection phase. Concretely, attention focus, goal orientation, goal setting, 
TABLE 1 | Compliance with EBI controls by the four intervention studies.

\begin{tabular}{|c|c|c|c|c|c|c|}
\hline $\mathrm{N}$ & Training intervention providers & Protocol & Pre-post measures & Number of groups & Recording of sessions & Interview check \\
\hline 4 & 1 & 2 & 3 & All studies used two groups & 4 & 1 \\
\hline
\end{tabular}

TABLE 2 | Mean effect sizes of SRL strategies.

\begin{tabular}{llllll}
\hline SRL strategies & $\boldsymbol{k}$ & Cohen's $\boldsymbol{d}$ & $\mathbf{9 5 \%} \mathbf{C l}$ & $\boldsymbol{Z}$ & $\boldsymbol{p}$ \\
\hline Attention focus & 8 & 0.537 & $0.189-0.885$ & 3.024 & 0.002 \\
Attribution & 8 & 0.272 & $-0.003-0.547$ & 1.942 & 0.052 \\
Goal orientation & 68 & 0.092 & $0.032-0.152$ & 2.997 & 0.003 \\
Goal setting & 4 & 0.474 & $0.311-0.636$ & 5.716 & 0.000 \\
Integrated & 5 & 1.018 & $0.284-1.752$ & 2.717 & 0.007 \\
Metacognitive & 43 & 0.388 & $0.316-0.460$ & 10.530 & 0.000 \\
monitoring & & & & & \\
Self-efficacy & 39 & 0.699 & $0.586-0.812$ & 12.133 & 0.000 \\
Self-evaluation & 13 & 0.717 & $0.430-1.004$ & 4.892 & 0.000 \\
Self-satisfaction & 3 & -0.033 & $-0.965-0.899$ & -0.070 & 0.944 \\
Task Interest/value & 9 & 0.405 & $0.022-0.787$ & 2.075 & 0.038 \\
Task strategies & 64 & 0.600 & $0.518-0.682$ & 14.379 & 0.000 \\
SRL (pooled) & 264 & 0.365 & $0.392-0.401$ & 19.945 & 0.000 \\
\hline
\end{tabular}

All the effect sizes were based on random effects analysis in the present study; Integrated stands for the independent samples that did not distinguish specific SRL strategy. In interpreting the effect size, we followed Rosenthal (1996)'s guidelines ( $d=0 \sim 0.29$, small; $d=0.30 \sim 0.79$, medium, $d=0.80 \sim 1.29$, large).

TABLE 3 | Mean effect sizes grouped according to the group of discipline.

\begin{tabular}{lcccccc}
\hline Discipline & $\boldsymbol{k}$ & Cohen's $\boldsymbol{d}$ & $\mathbf{9 5 \%} \mathbf{C l}$ & $\boldsymbol{Q}_{\boldsymbol{B}}$ & $\boldsymbol{d f}$ & $\boldsymbol{p}$ \\
\hline Science & 59 & 0.449 & $0.377-0.522$ & 22.412 & 2 & 0.000 \\
Language & 80 & 0.292 & $0.231-0.352$ & & & \\
Integrated & 125 & 0.592 & $0.465-0.719$ & & & \\
\hline
\end{tabular}

Integrated stands for the integration of mathematics performance and language performance.

and task interest/value were classified into the forethought phase. Task strategies, metacognitive monitoring, and attention focus were classified into the performance phase. Attribution, selfsatisfaction/affect, and self-evaluation were classified into the self-reflection phase. Table 4 showed that the differences among the three phases were significant $\left[Q_{B}(3)=22.025, p_{B}=0.000\right]$. The effect sizes of forethought phase, performance phase and self-reflection phase were $0.316,0.525$, and 0.465 .

\section{Moderator Analyses}

All independent samples were classified into three groups: elementary school, junior high school, and senior high school. Some independent samples included both elementary school students and junior high school students, or junior high school students and senior high school students, but did not specify the educational stage. These independent samples were not included in this moderator analysis. It was found that educational stage significantly moderated the relationship between SRL and
TABLE 4 | Mean effect sizes grouped according to the phases of SRL.

\begin{tabular}{lcccccc}
\hline SRL phases & $\boldsymbol{k}$ & Cohen's $\boldsymbol{d}$ & $\mathbf{9 5 \%} \mathbf{C l}$ & $\boldsymbol{Q}_{\boldsymbol{B}}$ & $\boldsymbol{d f}$ & $\boldsymbol{p}_{\boldsymbol{B}}$ \\
\hline Forethought & 119 & 0.316 & $0.245-0.386$ & 22.025 & 3 & 0.000 \\
Performance & 115 & 0.525 & $0.464-0.585$ & & & \\
Self-reflection & 25 & 0.465 & $0.261-0.669$ & & & \\
Integrated & 5 & 1.018 & $0.284-1.752$ & & & \\
\hline
\end{tabular}

Integrated stands for the independent samples that did not distinguish specific SRL strategy, these samples could not be classified to a specific phase of SRL.

TABLE 5 | Mean effect sizes grouped according to educational stage.

\begin{tabular}{lcccccc}
\hline Educational stage & $\boldsymbol{k}$ & Cohen's $\boldsymbol{d}$ & $\mathbf{9 5 \%} \mathbf{C l}$ & $\boldsymbol{Q}_{\boldsymbol{B}}$ & $\boldsymbol{d} \boldsymbol{f}$ & $\boldsymbol{p}_{\boldsymbol{B}}$ \\
\hline Elementary school & 19 & 0.164 & $0.038-0.289$ & 38.623 & 2 & 0.000 \\
Junior high school & 84 & 0.629 & $0.527-0.732$ & & & \\
Senior high school & 81 & 0.303 & $0.231-0.375$ & & &
\end{tabular}

Some independent samples included both elementary school students and junior high school students, or junior high school students and senior high school students, but did not distinguish specific educational stage. These independent samples were not included in this analysis.

academic performance $\left[Q_{B}(2)=38.623, p_{B}<0.001\right.$, See Table 5]. Specifically, the effect size of junior high school students (Cohen's $d=0.629$ ) was higher than that of senior high school students (Cohen's $d=0.303$ ) and elementary school students (Cohen's $d$ $=0.164)$.

The time span of the present meta-analysis ranged from 1998 to 2016. We examined the moderating effects of publication year and the proportion of female using meta-regression. From 1998 to 2016, the effect size of SRL gradually declined [Slope $=-0.020$, $Q_{\text {model }}(1)=147.470, p<0.001$. See Figure 2]. The moderating effect of the proportion of female was not found [Slope $e_{\text {FisherZ }}=$ $0.001, Q_{\text {model }}(1)=0.273, p=0.601 ;$ Slope $_{d}=0.001, Q_{\text {model }}(1)=$ $49.642, p<0.001]$.

\section{Publication Bias}

As is shown in Figure 3, there were almost no low accurate and high effect size studies in the funnel plot (bottom right), and the whole plot presents symmetry. In addition, the failsafe $N$ of the present study was 9,558. According to Rosenthal's (1996) suggestion, if the fail-safe $N$ is smaller than $5 k+10$, then the meta-analysis may have publication bias. In the presence of publication bias, studies are expected to be systematically missing in a manner that can be identified by the trim and fill analyses (Duvall and Tweedie, 2000). In the present meta-analysis, the trimmed studies were 0 . In summary, the results were not affected by publication bias. 


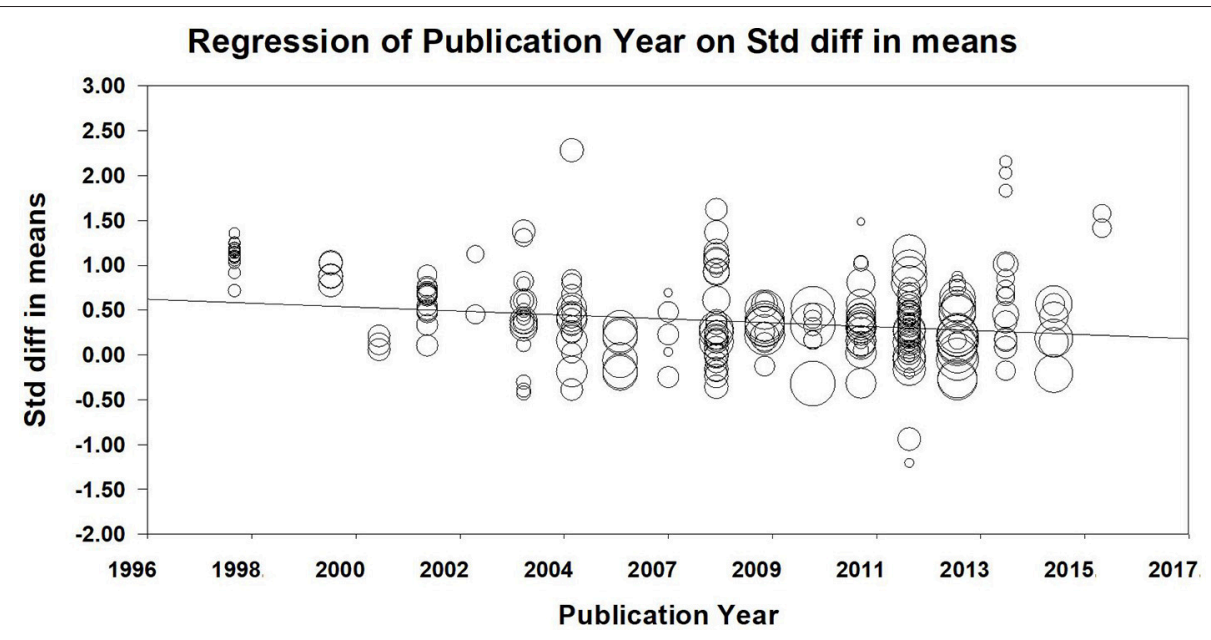

FIGURE 2 | The moderating effect of publication year.

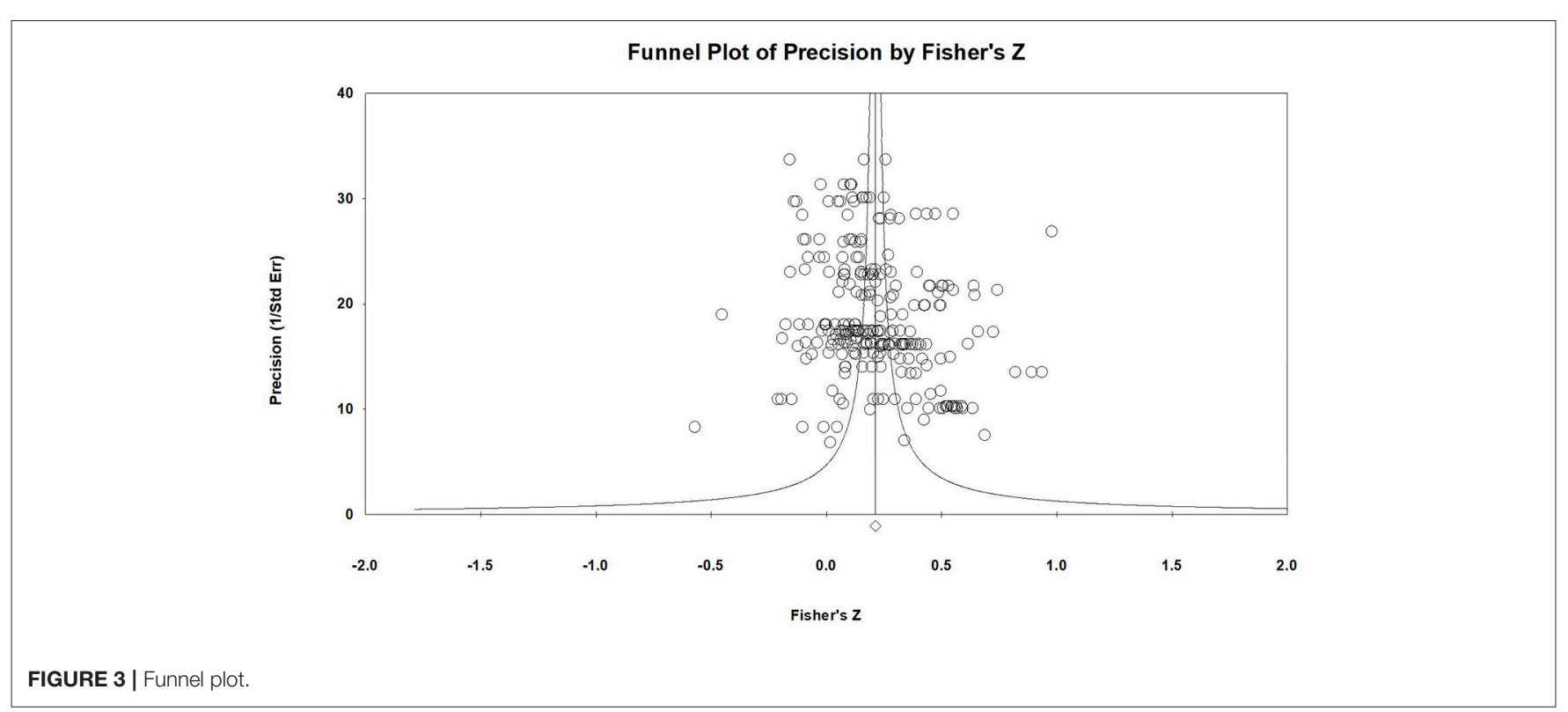

\section{DISCUSSION}

The present study used meta-analysis to examine data from 59 empirical studies (264 independent samples), covering 23,497 Chinese students, and tried to understand the relationship between SRL strategies and academic achievement in education in China. We examined the moderating effects of gender, grade, and year of publication. According to Rosenthal (1996)'s guidelines for effect size, analyses found that medium effect sizes of self-evaluation, self-efficacy, task strategies, which were relatively larger than other strategies. In contrast, the effect size of attribution and goal orientation were small but present. Basically, Hypothesis 1 was supported by these results. From the perspective of discipline, the effect size of SRL on the academic performance of science (medium) was higher than that of language (small). Moreover, the effect size of forethought phase was smaller than the other two phases, which suggested that performance and self-reflection were critical phases of SRL. This result lent support to Hypothesis 2. The moderating effects of grade and publication year were significant. The effect size of junior high school was larger than that of senior high school, and the effect size of elementary school was small, suggesting that Hypothesis 3 was supported. The moderating effect of publication year was found, with the relationship between SRL and academic gradually weakened from 1998 to 2016.

\section{The Effective SRL Strategies for Chinese Students}

We found that the effect sizes of self-efficacy, self-evaluation, and task strategies were relatively large, suggesting that these were important SRL strategies for Chinese students. Similar to 
the present study, Richardson et al. (2012) conducted a metaanalysis including 241 unique data sets from Europe and North America, and found that performance self-efficacy was strongly associated with GPA, comprising the largest effect observed. The effect size of strategic approach to learning was also larger than that of peer learning, learning goal orientation, and performance goal in their study. The meta-analysis of Dignath et al. (2008) also reported that students' self-efficacy represented the largest correlation with academic performance, and the effect sizes of goal setting, goal orientation, and attribution were relatively small. Task strategies (e.g., cognitive learning strategies) were also found to be effective for Korean learners in the previous meta-analytic research, which had a significantly large effect size (Kim et al., 2008a). Kizilcec et al. (2017) found strategic planning predicted attainment of personal course goals. These previous findings, together with results in the present meta-analysis, suggest that having knowledge and skill does not produce high-quality problem solving if learners lack the self-efficacy to use their personal resources. Task strategies help learning or problem solving by reducing a task to its essential parts and reorganizing them meaningfully. Self-evaluation compares selfmonitored outcomes with learners' goal or other standards. Each element may represent the essential function of its corresponding phase, and thus exhibit a large effect size in terms of its association to the academic achievement. Of course, this is not to say that goal orientation and attribution are unnecessary strategies. In fact, many definitions and theories have emphasized the importance of goal orientation and attribution (Cook and Artino, 2016; Latham et al., 2016). But in relative terms, self-efficacy, task strategies, and self-evaluation were more effective than goal and attribution. Therefore, in real teaching situations, educators should know how to improve students' self-efficacy, and teach them a few useful task strategies (e.g., cognitive strategies). Teachers also should guide students to evaluate their performance and to understand their strengths and weaknesses.

In addition, the present study found different effect sizes for the different academic fields (higher effects for science performance than for language), suggesting that SRL strategies were more effective for improving science performance. Lucangeli and Cornold (1997) suggested that metacognition seems to be very important to gain a better understanding of successful mathematical performance. The usage of SRL strategies may vary in different disciplines (Bembenutty et al., 2013). Wolters and Pintrich (1998) explored the use of SRL strategies of junior high school students in three academic subjects (i.e., mathematics, English, and social studies), and different subjects were reported to be different in value and interest for self-efficacy, academic tasks and test anxiety. The demand of cognitive ability in mathematics is higher than those of linguistics and social science (Loong, 2012), and cognitive ability closely related to SRL. Metallidou and Vlachou (2010) examined the SRL profile of fifth and sixth grade students who were differentiated in their task value beliefs in mathematics and language, and found that teachers' ratings for their students' knowledge and use of strategies significantly predicted students' mathematics performance, but not language performance. The results indicated a domain-specific aspect of SRL strategies.

\section{The Critical Phases of SRL for Chinese Students}

According to the phases and sub-processes of SRL, we classified all SRL strategies in empirical studies into forethought phase, performance phase, and self-reflection phase. The effect size of forethought phase (small) was lower than that of performance phase (medium) and self-reflection phase (medium), but there was no difference between performance phase and self-reflection phase.

From a theoretical perspective, researchers have different views in terms of whether SRL should contain the forethought phase (including motivation, goal setting, planning, and the like). For example, social cognitive view suggested that SRL is acquired through a triadic interaction between three characteristics: (1) self-observation (monitoring one's actions); (2) self-judgement (evaluation of one's performance); (3) self-reactions (one's response to performance outcomes), which did not involve the elements of motivation or planning (Broadbent and Poon, 2015). Miller and Brown (1991) proposed that SRL have seven procedures, covering information input, self-evaluation, instigation to change triggered by perceptions of discrepancy, search for ways to reduce discrepancy, planning for change, implementation of behavior change, and evaluation of progress toward a goal. Their model began to pay attention to goal setting and planning, but not motivation. Zimmerman's phases and sub-processes of SRL incorporated the elements of motivation, goal setting, and planning, all the three elements constituted the forethought phase. Thus, the disagreement between these theories focused on the forethought phase. Nevertheless, the aforementioned researchers all agreed that the cores of SRL are the process of self-monitoring and the execution of self-control behavior. The present meta-analysis found that the effect size of the forethought phase was lower than performance phase and self-reflection phase, suggesting that the core phases of SRL have a closer association with academic achievement. Based on the SRL theories and the results of the present study, the critical phases of SRL for Chinese students should be the performance phase and the self-reflection phase.

\section{The Moderating Effects of Education Stage and Publication Year}

Results of the present study indicates that the moderating effect of grade was significant, whereas the effect size of elementary school students was smaller than senior high school and junior high school. It could be because the development of motivation and self-efficacy of elementary school students is insufficient, and their SRL motivation, emotion and self-efficacy still stay fuzzy (Su et al., 2013). The effect size of junior high school students was two times larger than that of senior high school students. The cognitive development of junior high school students has already been in formal operational stage, and their abstract thinking ability is close to adult. Junior high school is also an important development stage of SRL (Nurjanah and Dahlan, 2018). However, we found that the relationship between SRL and academic achievement was decreasing from junior high school to senior high school. This might be because the speed of SRL 
development declines with age (Ji, 2005). The curriculum of senior high school is more difficult than junior high school, and the development of SRL also slows down. Moreover, there are many other factors that influence students' academic performance, such as teaching quality, curriculum difficulty, reasoning, problem solving, and peer relationships (Hernández et al., 2017; Llorca et al., 2017; Stadler et al., 2017). These factors may have enhancing effects on academic achievement, while the impact of SRL declines in high school.

From 1998 to 2016, the effect size of SRL gradually decreased, suggesting that the moderating effect of publication year was significant. One possible interpretation is the birth cohort effect (Xin and Chi, 2008). The earliest empirical study in the present meta-analysis was published in 1998, and the latest study was published in 2016. Middle school students in 1998 (post-80s) and in 2016 (post-00s) experienced different social and cultural environments. The most significant social change in China from 1998 to 2016 is probably the popularity of the Internet (CNNIC, 1996, 2016). The rapid and global expansion of the Internet has transformed the way our world operates and the way people think and interact (Gosling and Mason, 2015). Previous studies found that self-control (closely related to SRL) was associated with problematic Internet use among Chinese adolescents ( $\mathrm{He}$ et al., 2012; Sun et al., 2015; Mei et al., 2016). Lack of selfcontrol is one of the major characteristics of problematic use of Internet (Kim et al., 2008b). Internet is more accessible to post-00s (who are usually digital natives) than to post-80s (who are digital immigrants) during their high school study. Ransdell (2010) found that older cohorts of learners can be better online learners. Digital natives (i.e., Millennial students) showed poorer knowledge application skill and were more self-reliant than older students (Ransdell et al., 2011). Digital natives may use a resource of the online learning environment only if they deem it necessary; or else they may need extra encouragement to participate actively in a course and to exchange information with their peers (Ransdell et al., 2011). These evidences may help to explain the moderating effect of publication year in our study. However, the negative effect of Internet on self-control is only one of possible interpretations. It should be cautious when interpret the moderating effect of publication year on the association of SRL and academic achievement.

\section{Limitations and Directions for Future Research}

Some limitations to the current meta-analysis should be noted. First, the sample sizes of some SRL strategies were small, such as of self-satisfaction and goal setting, so we could not provide further discussion on these strategies. Moreover, few empirical studies reported social economic status (SES), which might be an important potential moderator (see, e.g., Pintrich, 2004) but cannot be analyzed in the present study.

Second, few SRL studies in China were conducted in online learning environments, hence the difference between traditional learning and online learning could not be analyzed. We also exclude the studies from special education. Compared to traditional classroom students, SRL ability to control, manage, and plan learning actions is particularly important to online learners (Ally, 2004). The online learning conditions were less likely to be instructor-directed than they were to be learnerdirected. Independent learning and collaborative learning are significant features of online learning (Adam et al., 2017). It is surmised that SRL is more important for online learning than traditional learning, which may yield different effect size.

Third, we did not evaluate the effects of the specific methodological controls of the included studies (i.e., studies conducted in real teaching situation) on the relationship between SRL and academic achievement. For example, the research design (e.g., experimental or quasi-experimental design) and fidelity of data collection may be different in the included studies. In addition, most empirical studies in the current meta-analysis were cross-sectional studies, whereas only four studies were based on intervention, which limited the generalizability of the current study to some extents. We evaluated the treatment fidelity of these intervention studies but found that few studies provided a rigorous treatment fidelity control. Despite these limitations, the current study provided an insight from Chinese scientific community to the field of SRL.

Future studies could examine the moderating effect of SES or other interesting variables. More SRL empirical studies in online learning are needed in China, so researchers could compare the difference effects between traditional learning and online learning on the relationship between SRL and academic performance. For example, Broadbent and Poon (2015) suggested that online learners should not dedicate time to using elaboration, rehearsal, and organizing when learning new curriculum as these SRL strategies may not increase the possibility of academic success. Specifically, massive open online courses (MOOCs) have attracted much attention from educators and students in the recent years, such courses may need high level SRL abilities to achieve academic success. Additionally, education technologies, such as educational games and intelligent tutoring system, develop fast in the last decade. From 2004 to 2016, the new media consortium predicted educational games, intelligent tutoring system, and virtual assistant could be important new technologies that impact higher education deeply. Future studies could analyze SRL strategies in educational games or explore whether immersion and other characteristics in games could promote students' SRL ability.

\section{CONCLUSIONS AND IMPLICATIONS}

The conclusions emerging from the present study are as follows: First, for Chinese elementary school students and high school students, self-efficacy, task strategies, and self-reflection turned to be better SRL strategies. Second, SRL strategies were more effective for science disciplines than for linguistics disciplines. Third, the performance phase and the self-reflection phase are critical phases of SRL in that they have a larger effect on academic achievement. Fourth, the effect size of junior high school was larger than that of senior high school and elementary school. Junior high school is probably the critical period of SRL development. Fifth, from 1998 to 2016, the effect 
size of SRL was gradually decreasing. Lastly, most empirical studies in the present meta-analysis were cross-sectional studies, whereas only four were intervention studies. More empiricalbased intervention studies may be necessary before generalizing the current conclusions to educational practice.

According to the present study, educators should improve students' self-efficacy, and teach them some sample task strategies. Teachers could encourage students to evaluate their own performance and to reflect their learning actions. It could be beneficial for teachers to know how to teach SRL at different educational levels and disciplines, because SRL works better at different educational levels and disciplines. At the same time, teachers need to receive training on SRL theory and models to understand how they can maximize their students' learning (Panadero, 2017). For example, new teachers need to receive pedagogical training for their future adaptation to the workplace. In-service teachers also need to receive training on SRL as they most probably did not receive any during their pre-service preparation (Moos and Ringdal, 2012). In addition, this study sheds light on how Chinese students use SRL in their learning. Self-efficacy, task strategies, and self-evaluation were the most important SRL strategies for Chinese students, whereas self-efficacy, grade goal setting, and effort regulation are particularly important for learners in Western countries (Richardson et al., 2012). It seems that students in China show a different SRL pattern, which may help to preliminarily explain the parity between the high performance (i.e., math)

\section{REFERENCES}

Adam, N. L., Alzahri, F. B., Soh, S. C., Bakar, N. A., and Kamal, N. A. M. (2017). "Self-regulated learning and online learning: a systematic review," in International Visual Informatics Conference (Cham: Springer), 143-154.

Ally, M. (2004). "Foundation of educational theory for online learning," in The Theory and Practice of Online Learning, ed T. Anderson (Edmonton, CA: Athabasca University Press), 15-44. Available online at: http:// publicservicesalliance.org/wp-content/uploads/2016/09/Theory-andPractice-of-Online-Learning.pdf

*An, M., and Yu, J. Y. (2009). The relationship between metacognitive monitoring and academic performance of secondary school students. Inner Mong. Educ. 2009, 11-13. doi: 10.1177/0013164492052002017

Baumeister, R. F., Heatherton, T. F., and Tice, D. M. (1994). Losing Control: How and Why People Fail at Self-Regulation. San Diego, CA: Academic Press.

Bembenutty, H., Cleary, T. J., and Kitsantas, A. (eds.). (2013). Applications of Self-Regulated Learning Across Diverse Disciplines: A Tribute to Barry J. Zimmerman. Charlotte, NC: IAP Information Age Publishing.

Blumenfeld, P. C., Pintrich, P. R., Meece, J., and Wessels, K. (1982). The formation and role of self-perception of ability in elementary classrooms. Element. Sch. J. 82, 401-420. doi: 10.1086/461278

Borenstein, M., Hedges, L., Higgins, J., and Rothstein, H. (2009). Introduction to Meta-Analysis. Chichester, UK: John Wiley and Sons, Ltd.

Borenstein, M., Hedges, L., Higgins, J., and Rothstein, H. (2010). A basic introduction to fixed-effect and random-effects models for meta-analysis. Res. Synth. Methods 1, 97-111. doi: 10.1002/jrsm.12

Borenstein, M., Rothstein, D., and Cohen, J. (2005). Comprehensive MetaAnalysis: A Computer Program for Research Synthesis [Computer Software]. Englewood, NJ: Biostat.

Broadbent, J., and Poon, L. W. (2015). Self-regulated learning strategies \& academic achievement in online higher education learning and a lack of advanced teaching methods in China (Leung et al., 2006). However, there is still need for experimental and longitudinal studies exploring the answers for this question in the future.

\section{AUTHOR CONTRIBUTIONS}

JL searched for references, coded and analyzed the data, and prepared the manuscript. HY coded the data and helped prepare the manuscript. YT designed the study and prepared the manuscript. $\mathrm{ZZ}$ and $\mathrm{XH}$ supervised the whole research process and proofread the manuscript.

\section{FUNDING}

This research was financially supported by doctoral research funds of Central China Normal University from the colleges' basic research and operation of Ministry of Education [\#2017YBZZ083] and the Research Program Funds of the Collaborative Innovation Center of Assessment toward Basic Education Quality [\#2016-04-011-BZK01].

\section{SUPPLEMENTARY MATERIAL}

The Supplementary Material for this article can be found online at: https://www.frontiersin.org/articles/10.3389/fpsyg. 2018.02434/full\#supplementary-material environments: a systematic review. Internet Higher Educ. 27, 1-13. doi: 10.1016/j.iheduc.2015.04.007

Canedo-García, A., García-Sánchez, J.-N., and Pacheco-Sanz, D.-I. (2017). A systematic review of the effectiveness of intergenerational programs. Front. Psychol. 8:1882. doi: 10.3389/fpsyg.2017.01882

*Cao, L. R., and Cao, X. H. (2004). The influence of time management disposition, cognitive style, and meta-worry on the high school students' achievement. Chin. J. Ergon. 10, 13-15.

*Chang, H. Q., and Pan, Y. (2013). A correlational study of time management and academic performance in secondary vocational school students. Academy 2013, 175-176.

*Chen, C., Gong, S. Y., and Chai, X. Y. (2013). Parent goals, classroom goal structures, personal achievement goal orientations and academic performance for middle school students. Psychol. Res. 6, 78-84.

*Chen, J. (2009). The relationship between goal orientation, seeking help and academic performance of high school students. Shanghai Youth 2009, 21-23.

*Chen, J. J., and Li, S. F. (2012). The paths of junior school students' achievement attribution and academic emotions forecasting their academic achievement. Chin. J. Clin. Psychol. 20, 392-394.

*Chen, L. J. (2011). Relationship among achievement goal orientation, self-efficacy and academic performance of high school students. China J. Health Psychol. 19, 718-719.

*Chen, M. G., and Hu, Z. B. (2008). Research on the relationships among goal orientation, learning strategy, attribution and academic performance in high school students. Psychol. Explorat. 28, 58-62.

*Chen, X. Q., Zhang, Y. X., Jiang, Q., and Meng, X. Q. (2014). The effects of metacognitive strategy training on English learning in high school students. Middle Sch. Stud. 2014, 109-110.

*Chi, Y. K., and Zhou, G. T. (2003). The effects of perceived pedagogical caring and self-regulate learning on academic achievement. J. Guangdong Educ. Inst. 23, 47-51. 
Cleary, T., and Zimmerman, B. J. (2010). Self-regulation differences during athletic practice by experts, non-experts, and novices. J. Appl. Sport Psychol. 13, 61-82.

Cleary, T., Zimmerman, B. J., and Keating, T. (2006). Training physical education students to self-regulate during basketball free-throw practice. Res. Q. Exerc. Sport 77, 251-262. doi: 10.1080/02701367.2006.105 99358

CNNIC (1996). The Statistics Report the Development of China Internet Network. (In Chinese). Beijing: China Internet Network Information Center (CNNIC).

CNNIC (2016). The 38th Statistics Report the Development of China Internet Network. (In Chinese). Beijing: Internet Network Information Center (CNNIC). Available online at: http://www.cnnic.net.cn/hlwfzyj/hlwxzbg/ hlwtjbg/201608/P020160803367337470363.pdf

Cook, D. A., and Artino, A. R. (2016). Motivation to learn: an overview of contemporary theories. Med. Educ. 50, 997-1014. doi: 10.1111/medu.13074

${ }^{*}$ Cui, N., Liu, Y. Y., and Gao, B. Y. (2008). The relationship between achievement goal, examining anxiety and performance in senior students. China J. Health Psychol. 16, 436-438.

Díaz-Prieto, C., and García-Sánchez, J. N. (2015). Identification of relevant elements for promoting effective interventions in old age. J. Psychodidact. 21, 157-173. doi: 10.1387/RevPsicodidact.13854

Dignath, C., Buettner, G., and Langfeldt, P. H. (2008). How can primary school students learn self-regulated learning strategies most effectively? A metaanalysis on self-regulation training programmes. Educ. Res. Rev. 3, 101-129. doi: 10.1016/j.edurev.2008.02.003

Doménechbetoret, F., Abellánroselló, L., and Gómezartiga, A. (2017). Selfefficacy, satisfaction, and academic achievement: the mediator role of students' expectancy-value beliefs. Front. Psychol. 8:1193. doi: 10.3389/fpsyg.2017.01193

Duvall, S., and Tweedie, R. (2000). A nonparametric "trim and fill" method of accounting for publication bias in meta-analysis. J. Am. Stat. Assoc. 95, 89-99. doi: $10.2307 / 2669529$

Eccles, J., Wigfield, A., Harold, R. D., and Blumenfeld, P. (1993). Age and gender differences in children's self and task perceptions during elementary school. Child Dev. 64, 830-847.

EU Council (2002). "Council resolution 27 June 2002 on lifelong learning," in Official Journal of the European Communities, C 163, 09 July 2002. Available online at: https://publications.europa.eu/en/publication-detail/-/publication/ ab179471-65eb-4c7b-b7d6-2d956a4fcc17/language-en

Everaert, P., Opdecam, E., and Maussen, S. (2017). The relationship between motivation, learning approaches, academic performance and time spent. Account. Educ. 26, 78-107. doi: 10.1080/09639284.2016.1274911

Fernandez-Rio, J., Cecchini, J. A., Méndez-Gimenez, A., Mendez-Alonso, D., and Prieto, J. A. (2017). Self-regulation, cooperative learning, and academic self-efficacy: interactions to prevent school failure. Front. Psychol. 8:22. doi: 10.3389/fpsyg.2017.00022

${ }^{*}$ Gao, R. C., Liu, R. D., Wang, D., He, M. J., and Yuan, Z. (2011). Types of selfregulated learning of junior middle school students and their influence on learning. Psychol. Dev. Educ. 2011, 76-82.

*Ge, M. G., and Jin, Y. (2005). A study on the relationships between English learning strategies and academic achievement of middle school students. Psychol. Sci. 28, 451-453.

Gosling, S. D., and Mason, W. (2015). Internet research in psychology. Annu. Rev. Psychol. 66, 877-902. doi: 10.1146/annurev-psych-010814-015321

${ }^{*}$ Gu, S. H., Xin, T., and Li, H. (1998). A correlational study of attribution, learning strategy, and academic achievement in junior high school students. Psychol. Dev. Educ. 1998, 21-25.

*Guo, W. (2008). A study on the relationship between the factors of personality and academic results of middle school students. J. Fu Qing Branch Fujian Norm. Univ. 2008, 46-50.

*Han, X. H., Zheng, L. N., and Liu, R. J. (2015). The mediation effect of selfregulated learning in parent involvement and academic achievement in higher elementary school students. J. Liaocheng Univ. 28, 74-78.

He, C., Xia, M., Jiang, G. R., and Wei, H. (2012). Mediation role of self-control between Internet game addiction and self-esteem. Chin. J. Clin. Psychol. 20, 58-60.

Hedges, L. V., and Vevea, J. L. (1998). Fixed and random effects models in meta-analysis. Psychol. Methods 3, 486-504. doi: 10.1037/1082-989X.3.4.486

Hernández, M. M., Valiente, C., Eisenberg, N., Berger, R. H., Spinrad, T. L., and Vanschyndel, S. K., et al. (2017). Elementary students' effortful control and academic achievement: the mediating role of teacher-student relationship quality. Early Child. Res. Q. 40, 98-109.

Hyde, J. S., Lindberg, S. M., Linn, M. C., Ellis, A. B., and Williams, C. C. (2008). Gender similarities characterize math performance. Science 321, 494-495. doi: $10.1126 /$ science. 1160364

Jerrim, J., and Shure, N. (2016). Achievement of 15-Year-Olds in England: PISA 2015 National Report. Available online at: https://assets.publishing.service.gov. uk/government/uploads/system/uploads/attachment_data/file/574925/PISA2015_England_Report.pdf

*Ji, H. Y. (2005). The relationship between middle school students' self-regulated learning development and academic performance. J. Chuzhou Univ. 7, 97-100.

*Jiang, B., and Tan, D. L. (2007). The effects of peer learning on academic performance and social development of junior high school students. J. Tianjin Acad. Educ. Sci. 2007, 33-35.

*Jiang, J. C., and Liu, H. S. (2005). Research on the relationship between the achievement goal orientation and the learning strategy \& the academic performance. Psychol. Dev. Educ. 2005, 56-61.

* Jiang, M. R., Song, H. S., and Zhang, L. (2014). Research on correlation between self-evaluation ability and academic achievement. J. Kashgar Teach. Coll. 35, 66-68.

*Jiang, Y. Z., and Zhang, H. Z. (2012). A correlational research of self-esteem, selfefficacy and academic performance in junior high school students. Educ. Meas. Evaluat. 2012, 43-50.

*Jiao, C. Z. (2008). Relationship of mathematic interest self-efficacy and mathematic achievement of junior school students. J. Math. Educ. 17, 44-46.

Jiao, X. Y., and Gai, X. S. (2011). The gender differences of self-regulation in children. Chin. J. Spec. Educ. 2011, 79-83.

*Jin, G. Y., Gao, J. J., and Chen, Y., W. (2012). How learning strategy and self-efficacy predict achievement in different situations. Journal of Zhejiang University 39, 231-238.

Kim, D., Kim, B., Lee, K., Park, J., Hong, S., and Kim, H. (2008a). Effects of cognitive learning strategies for Korean Learners: a meta-analysis. Asia Pacific Educ. Rev. 9, 409-422. doi: 10.1007/BF03025659

Kim, E. J., Namkoong, K., Ku, T., and Kim, S. J. (2008b). The relationship between online game addiction and aggression, self-control and narcissistic personality traits. Eur. Psychiatry 23, 212-218. doi: 10.1016/j.eurpsy.2007. 10.010

Kitsantas, A., and Zimmerman, B. J. (2002). Comparing self-regulatory processes among novice, non-expert, and expert volleyball players: a microanalytic study. J. Appl. Sport Psychol. 14, 91-105. doi: 10.1080/104132002529 07761

Kizilcec, R. F., Pérez-Sanagustín, M., and Maldonado, J. J. (2017). Self-regulated learning strategies predict learner behavior and goal attainment in Massive Open Online Courses. Comput. Educ. 104, 18-33. doi: 10.1016/j.compedu.2016.10.001

Latham, G., Seijts, G., and Slocum, J. (2016). The goal setting and goal orientation labyrinth: effective ways for increasing employee performance. Organ. Dyn. 45, 271-277. doi: 10.13140/RG.2.1.2692.4888

*Lei, L., Wang, L., and Culjak, T. (2001). The role of goal-orientation in selfregulated learning. Acta Psychol. Sin. 33, 349-353.

Leung, F. K. S., Graf, K. D., and Lopez-Real, F. J. (eds.). (2006). Mathematics Education in Different Cultural Traditions-A Comparative Study of East Asia and the West: The 13th ICMI Study, Vol. 9. Boston, MA: Springer Science \& Business Media.

${ }^{*} \mathrm{Li}$, B., and Yang, J. L. (2004). Relations of time management disposition, selfefficiency, attribution of junior school students to academic achievement. Psychol. Explor. 24, 67-71.

${ }^{*} \mathrm{Li}, \mathrm{H}$. X., Lin, X., and Si, J. W. (2015). The relationship between time management disposition and academic achievement in boarding primary school students: The mediating role of self-regulate learning. Psychol. Res. 8, 90-96.

Li, J., Pang, B., Zhang, B., and Du, H. (2011a). Self-regulation: From basic theories to applications. J. Beijing Norm. Univ. 2011, 5-13. doi: 10.1190/1.3659042

*Li, J., Zhang, J., and Zhu, L. Q. (2011b). The development of middle school students' self-evaluation and its relationship with academic achievement. Psychol. Sci. 34, 619-624.

*Li, J. D., An, M., He, T. T., and Ge, G. Y. (2009). The relationships among psychological resilience, meta-cognition, self-efficacy, test anxiety and academic performance in middle school students. Exam. Wkly 2009, 13-14. 
${ }^{*} \mathrm{Li}, \mathrm{S}$., and Yu, X. X. (2002). The effects of self-concept, self-monitoring and learning strategy on academic achievement in middle school students. J. Ningbo Univ. 24, 18-22.

* Li, X. Y., and Li, J. W. (2004). Relative study on middle school students' mathematical achievement motivation self-regulated learning and mathematical achievement. J. Math. Educ. 13, 52-54.

${ }^{*} \mathrm{Li}$, Z. X., and Hu, Y. R. (2014). The relationship between goal orientation and academic achievement in junior high school students: the mediation of self-efficacy. J. Hubei Univ. Sci. Technol. 34, 123-125.

*Liu, J. L., and Liu, S. X. (2016). The relationship between learning attitude and academic achievement of junior middle school students. J. Gannan Norm. Univ. 2016, 106-109.

*Liu, J. X., Xin, T., Huang, G. Q., and Shen, J. L. (2000). A correlational study of learning motivation, learning 中 strategy and academic performance in middle school students. Theory Pract. Educ. 20, 54-58.

Liu, R. D., and Chen, Q. (2000). The mediate influence of on-line self-regulated learning activities on the learning outcomes in different learning situations. Acta Psychol. Sin. 32, 197-202.

*Liu, T. (2015). On relationship between academic adaptability, academic selfefficacy and academic performance of junior middle school students. J. Baicheng Norm. Univ. 29, 62-66.

Llorca, A., Cristina, M. R., and Malonda, E. (2017). Parenting, peer relationships, academic self-efficacy, and academic achievement: direct and mediating effects. Front. Psychol. 8:2120. doi: 10.3389/fpsyg.2017.02120

Loong, E. T. (2012). Self-regulated learning strategies and their effects on math performance of pre-university international students in Malaysia. J. Int. Educ. Res. 8, 223-232. doi: 10.19030/jier.v8i3.7104

Lucangeli, D., and Cornold, C. (1997). Mathematics and metacognition: what is the nature of the relationship? Math. Cogn. 3, 121-139. doi: $10.1080 / 135467997387443$

Mei, S., Yau, Y. H. C., Chai, J., Guo, J., and Potenza, M. N. (2016). Problematic internet use, well-being, self-esteem and self-control: data from a high-school survey in China. Addict. Behav. 61, 74-79. doi: 10.1016/j.addbeh.2016.05.009

Metallidou, P., and Vlachou, A. (2010). Children's self-regulated learning profile in language and mathematics: the role of task value beliefs. Psychol. Sch. 47, 776-788. doi: $10.1002 /$ pits.20503

Miller, W. R., and Brown, J. M. (1991). "Self-regulation as a conceptual basis for the prevention and treatment of addictive behaviors," in Self-Control and the Addictive Behaviours, N. Hether, W. R. Miller, and J. Greely (Sydney: Maxwell Macmillan), 3-79.

Mischel, W., Ayduk, O., Berman, M. G., Casey, B. J., Gotlib, I. H., Jonides, J., et al. (2010). Willpower' over the life span: decomposing self-regulation. Soc. Cogn. Affect. Neurosci. 6, 252-256. doi: 10.1093/scan/nsq081

Moos, D. C., and Ringdal, A. (2012). Self-regulated learning in the classroom: a literature review on the teacher's role. Educ. Res. Int. 2012:423284. doi: $10.1155 / 2012 / 423284$

Mullis, I. V., Martin, M. O., Gonzalez, E. J., and Chrostowski, S. J. (2004). TIMSS 2003 International Mathematics Report: Findings from IEA's Trends in International Mathematics and Science Study at the Fourth and Eighth Grades. TIMSS \& PIRLS International Study Center. Available online at: https://files. eric.ed.gov/fulltext/ED494651.pdf

Mullis, I. V., Martin, M. O., Gonzalez, E. J., Gregory, K. D., Garden, R. A., O'Connor, K. M., et al. (2000). TIMSS 1999 International Mathematics Report. Available online at: https://timss.bc.edu/timss1999i/math_achievement_ report.html

National Research Council (1992). Combining Information: Statistical Issues and Opportunities for Research. Washington, DC: National Academy Press.

Nurjanah, and Dahlan, J. A. (2018). Improving self-regulated learning junior high school students through computer-based learning. J. Phys. 1013:012126. doi: 10.1088/1742-6596/1013/1/012126

Ortiz, A, E., and Bornacelly, I. (2017). CIMA Brief\#5: Are Girls Performing Better Than Boys in Education? Available online at: https://publications.iadb.org/ bitstream/handle/11319/8830/CIMA-Brief-5-Are-girls-performing-betterthan-boys-in-education.pdf? sequence $=3$ \& is Allowed $=y$

Panadero, E. (2017). A review of self-regulated learning: six models and four directions for research. Front. Psychol. 8:422. doi: 10.3389/fpsyg.2017.00422

Paris, S. G., and Newman, R. S. (1990). Developmental aspects of self-regulated learning. Educ. Psychol. 25, 85-102. doi: 10.1207/s15326985ep2501_7
Paris, S. G., and Paris, A. H. (2001). Classroom applications of research on self-regulated learning. Educ. Psychol. 36, 89-101. doi: 10.1207/S15326985EP3602_4

Pascoe, L., Spencer-Smith, M., Giallo, R., Seal, M. L., Georgiou-Karistianis, N., Nosarti, C., et al. (2018). Intrinsic motivation and academic performance in school-age children born extremely preterm: the contribution of working memory. Learn. Individ. Differ. 64, 22-32. doi: 10.1016/j.lindif.2018.04.005

Perry, N. E., Phillips, L., and Dowler, J. (2004). Examining features of tasks and their potential to promote self-regulated learning. Teach. Coll. Rec. 106, 1854-1878. doi: 10.1111/j.1467-9620.2004.00408.x

Perry, N. E., VanderKamp, K. O., Mercer, L. K., and Nordby, C. J. (2002), Investigating teacher-student interactions that foster self-regulated learning. Educ. Psychol. 37, 5-15. doi: 10.1207/S15326985EP3701_2

Pintrich, P. R. (1999). The role of motivation in promoting and sustaining self-regulated learning. Int. J. Educ. Res. 31, 459-470. doi: 10.1016/S0883-0355(99)00015-4

Pintrich, P. R. (2004). A conceptual framework for assessing motivation and self-regulated learning in college students. Educ. Psychol. Rev. 16, 385-407. doi: 10.1007/s10648-004-0006-X

*Ran, R., Huang, Y. Q., and Yu, J. (2012). The relationship between self-efficacy and academic achievement of junior high school students in the mainland Tibetan class. J. Jiangsu Teach. Univ. Technol. 18, 79-83.

Ransdell, S. (2010). Motivation, reasoning, and online activity among health science students. Comput. Human Behav. 26, 70-73. doi: $10.1016 /$ j.chb.2009.09.002

Ransdell, S., Kent, B., Gaillard-Kenney, S., and Long, J. (2011). Digital immigrants fare better than digital natives due to social reliance. Br. J. Educ. Technol. 42, 931-938. doi: 10.1111/j.1467-8535.2010.01137.x

Richardson, M., Abraham, C., and Bond, R. (2012). Psychological correlates of university students' academic performance: a systematic review and metaanalysis. Psychol. Bull. 138, 353-387. doi: 10.1037/a0026838

Rosenthal, J. A. (1996). Qualitative descriptors of strength of association and effect size. J. Soc. Serv. Res. 21, 37-59. doi: 10.1300/J079v21n04_02

Rothstein, H. R., Sutton, A. J., and Borenstein, M. (eds.). (2006). Publication Bias in MetA-Analysis: Prevention, Assessment and Adjustments. Chichester, UK: John Wiley \& Sons Ltd.

Royer, J. M., and Walles, R. (2007). "Influences of gender, motivation and socioeconomic status on mathematics performance," in Why is Math so Hard for Some Children, eds D. B. Berch and M. Mazzocco (Baltimore, MD: Brookes), 349-368.

Sadati, S., and Simin, S. (2017). The relationship between metacognitive and selfregulated learning strategies with learners' L2 learning achievement. Int. J. Res. Stud. Lang. Learn. 5, 97-106. doi: 10.5861/ijrsll.2015.1267

Sahdan, S. B., and Abidin, N. A. B. Z. (2017). Self-regulated learning: a literature review for 21st century learning technology. Adv. Sci. Lett. 23, 912-915. doi: $10.1166 /$ asl.2017.7435

Schneider, W., and Lockl, K. (2002). "The development of metacognitive knowledge in children and adolescents." in Applied Metacognition, eds T. J. Perfect and B. L. Schwartz (Cambridge: University Press), 224-257.

Silverman, I. W. (2002). Gender differences in resistance to temptation: theories and evidence. Dev. Rev. 23, 219-259. doi: 10.1016/S0273-2297(03)00012-1

Stadler, M., Becker, N., Schult, J., Niepel, C., Spinath, F. M., Sparfeldt, J. R., et al. (2017). The logic of success: the relation between complex problem-solving skills and university achievement. Higher Educ. 76, 1-15. doi: $10.1007 /$ s10734-017-0189-y

Sternberg, R. J., and Williams, W. M. (2010). Educational Psychology. New York, NY: Pearson.

Stipek, D., and Tannatt, L. (1984). Children's judgements of their own and their peers' academic competence. J. Educ. Psychol. 76, 75-84.

Street, E. K., Malmberg, L., and Stylianides, J. G. (2017). Level, strength, and facetspecific self-efficacy in mathematics test performance. ZDM Math. Educ. 49, 379-395. doi: 10.1007/s11858-017-0833-0

Su, D., Song, J., and Fang, P. (2013). Self-regulated study of students from primary to high school: a developmental study. Stud. Psychol. Behav. 11, 490-496.

Sun, X. J., Zhao, J., Zhou, Z. K., Chen, W., and Bao, N. (2015). Mediation role of self-control in Internet use between time management disposition and pathological Internet use. Stud. Psychol. Behav. 13, $410-413$. 
*Wang, J. X., and Zhang, K. (2008). Attribution, self-efficacy, examination anxiety and performance of middle school student. Psychol. Res. 1, 89-91.

*Wang, L. J. (2013). Research on the correlation among middle school students' cognitive interference, achievement goal orientation and their academic performance. J. Zhongzhou Univ. 30, 98-104.

*Wang, Y. X., Cao, Y. T., and Tang, X. L. (2013). Senior middle school students' academic self-efficacy and related study of its relationship with academic achievements. J. Weinan Norm. Univ. 28, 141-144.

*Wang, Y. Z., Wang, S. Z., and Ou, Y. L. (2005). A study on the relationships of academic achievements, academic self-efficacy and intrinsic motivations among middle school students in poverty-stricken areas. Psychol. Sci. 28, $826-829$.

*Wei, X. C., and Zhang, Y. F. (2014). A correlational study on the relationships among self-efficacy, attribution, and Chinese academic performance in middle school students. Res. Teach. 37, 117-120.

Wolters, C. A., and Pintrich, P. R. (1998). Contextual differences in student motivation and self-regulated learning in mathematics, english, and social studies classrooms. Instr. Sci. 26, 27-47. doi: 10.1023/A:10030359 29216

*Wu, J. Y., Hu, X. L., and Peng, J. S. (2011). Relationship between self-efficacy and academic performance of physics of senior middle school students. Educ. Teach. Res. 25, 122-125.

Xin, Z. Q., and Chi, L. P. (2008). Cross-temporal meta-analysis:discovering psychological development during social changes. J. East China Norm. Univ. $26,44-51$.

*Xu, J. B. (2012). An experimental study of self-regulated learning in English reading education. Engl. Teach. Res. Pract. Prim. Second. Sch. 2012, 80-83.

${ }^{*} \mathrm{Xu}$, L. L., and Guo, W. (2007). The relationships among goal orientation, English learning strategies and academic performance in senior high school students. Data Culture Educ. 2007, 146-147.

*Yao, C. L. (2013). A correlational research of English learning anxiety, metacognition and academic performance. Teach. Pract. 2013, 54-60.

*Yao, D. W., Yan, L. F., Musa, A., and Liu, G. (2011). Relationship among cognitive styles, learning motivation and strategies of junior students and the effect to school work achievement. Psychol. Res. 4, 92-96.

*Yuan, L. X. (2005). The study of relationship between achievement goals, selfefficacy, learning strategies and academic achievement. J. Guangdong Educ. Inst. $25,58-61$.

*Zhang, F., and Liu, C. (2012). Self-control of time management and academic performance of middle school students. Psychol. Res. 5, 79-84.

*Zhang, J. (2011). The relationships among English learning strategies, selfefficacy, and academic performance in senior high school students. J. Basic Engl. Educ. 13, 42-47.

*Zhang, J., Liu, J. W., and Wu, Q. L. (2012a). A model of goal achievement orientation, self-efficacy, metacognition and academic performance. Psychol. Res. 5, 85-88.

${ }^{*}$ References marked with an asterisk indicate studies included in the metaanalysis.
*Zhang, J. X., and Zhang, C. (2008). A comparative study of meta-cognition and academic performance in primary and secondary school students. Data Cult. Educ. 2008, 95-97.

*Zhang, L., and You, Z. Q. (2014). The effects of rural lower secondary school students' belief in a just world, gratitude and time management on their academic achievements. Chin. J. Spec. Educ. 2014, 62-67.

* Zhang, L. H., Sun, X. J., and Feng, J. Y. (2011). The associations between monitoring, reading performance, and self-regulated learning of elementary school students. Mental Health Primary Second. Sch. 2011, 10-12.

Zhang, P., Liang, Z. B., Chen, H. C., and Zhang, G. Z. (2012b). The stability and gender difference of children's self-control from 2 to 11 years old. Psychol. Dev. Educ. 2012, 463-470.

*Zhang, W. (2013). Related research on students' self-efficacy and mathematic achievement in the third grade of junior middle school. J. Nanchang Teach. Coll. 28, 109-110.

*Zhang, W. H., and Shen, J. L. (2006). A study on the relationship between middle school students' academic stress, achievement goal orientation and academic achievement. J. Southwest China Norm. Univ. 32, 95-98.

*Zhang, X. H., and Gao, C. Y. (2010). A correlational study of gender difference, meta-cognition and English listening performance. Overseas Engl. 2010, 339-340.

*Zhang, X. X., and Yang, Y. X. (2012). A correlational study of junior high school students' self-esteem, achievement goal and academic performance. Educ. Forum 2012, 155-156.

*Zhang, Y., and Li, Q. W. (2010). The relationships of junior high school students' teacher-student relationships, attribution style and achievement goal orientation with academic achievement. Psychol. Sci. 33, 785-788.

*Zhang, Y. F., Wei, X. C., and He, H. (2014). A comparative study of attribution style and academic performance in senior high school students. Educ. Pract. Res. 2014, 5-9.

*Zhou, L. H., and Liu, Y. (2012). The impact factors of physic academic performance of senior high school students. Phys. Educ. Res. Curric. Theory 2012, 105-107.

Zimmerman, B. J. (1990). Self-regulated learning and academic achievement: an overview. Educ. Psychol. 25, 3-17. doi: 10.1207/s15326985ep2501_2

Zimmerman, B. J. (2008). Investigating self-regulation and motivation: historical background, methodological developments, and future prospects. Am. Educ. Res. J. 45, 166-183. doi: 10.3102/0002831207312909

Conflict of Interest Statement: The authors declare that the research was conducted in the absence of any commercial or financial relationships that could be construed as a potential conflict of interest.

Copyright (c) $2018 \mathrm{Li}$, Ye, Tang, Zhou and Hu. This is an open-access article distributed under the terms of the Creative Commons Attribution License (CC BY). The use, distribution or reproduction in other forums is permitted, provided the original author(s) and the copyright owner(s) are credited and that the original publication in this journal is cited, in accordance with accepted academic practice. No use, distribution or reproduction is permitted which does not comply with these terms. 